\title{
The devil is in the domain: understanding protein recognition of multiple RNA targets
}

Glen R. Gronland ${ }^{1}$ and Andres Ramos ${ }^{1}$

1. Institute of Structural and Molecular Biology, University College London, London WC1E 6XA, UK

Correspondence to Andres Ramos: a.ramos@ucl.ac.uk

\begin{abstract}
:
RNA regulation provides a finely-tuned and highly-coordinated control of gene expression. Regulation is mediated by hundreds to thousands of multi-functional RNA-binding proteins which often interact with large sets of RNAs. In this brief review, we focus on recent work that highlights how the proteins use multiple RNA-binding domains to interact selectively with the different RNA targets. De-convoluting the molecular complexity of the RNA regulatory network is essential to understanding cell differentiation and function, and requires accurate models for protein-RNA recognition and protein target selectivity. We discuss that the structural and molecular understanding of the key determinant of recognition, together with the availability of methods to examine protein-RNA interactions at the transcriptome level, may provide an avenue to establish these models.
\end{abstract}

\author{
Abbreviations list: \\ CSD, Cold shock domain \\ dsRBD, Double-stranded RNA-binding domain \\ $\mathrm{KH}$, hnRNP K-homology \\ mRNA, Messenger RNA \\ ncRNA, Non-coding RNA \\ RBD, RNA-binding domain \\ RBP, RNA-binding protein \\ RRM, RNA-recognition motif \\ $\mathrm{ZnF}$, Zinc finger
}




\section{Introduction}

The combined regulation of the various steps in the metabolism and transport of messenger RNAs (mRNAs) and non-coding RNAs (ncRNAs) multiplies genomic potential, and allows cellular differentiation and the development of complex organisms. In the cell, functional RNAs are associated with a fluctuating assortment of multi-domain RNA-binding proteins (RBPs), forming integrated complexes, whose composition directs the fate of the transcript $t^{1,2}$. Individual RBPs bind multiple targets, and play a number of roles in RNA regulation (Figure 1). RBPs function as chaperonins, as well as regulators of the interactions between mRNAs and the large cellular machines for RNA processing, editing, transport, degradation, localisation and (for mRNAs) translation ${ }^{3,4,5}$. RBPs also mediate the communication between RNA regulation and key signalling networks via the post-translational regulatory events ${ }^{6}$. Below we discuss that the recognition of different RNA targets by the same protein is achieved via the target-dependent use of multiple RNA-binding domains. We also briefly discuss the implication of target-specific recognition for our understanding of protein-RNA regulatory networks.

\section{RNA regulation is mediated by multi-functional RNA-binding proteins that bind ensembles of RNA targets}

The RNA-binding proteins modulating RNA metabolism and transport bind and regulate many different RNA targets. Current estimates of the number of mRNA-binding proteins in the cell vary between one and two thousand, of which a few hundred have been validated functionally ${ }^{7}$. Considering that the majority of proteins known to bind and regulate ncRNAs are also mRNAbinding proteins, these estimates likely provide a reliable ballpark figure for the total number of RNA-binding proteins in a cell. On the contrary, a typical human cell contains ten to twenty thousand different mRNAs, each with many recognition sites for different RBPs ${ }^{8}$. Furthermore, a cell contains a large number of non-coding, but functional RNA molecules, which are also bound by proteins. The much larger number of RNA targets implies that many RBPs bind (and regulate) a large ensemble of RNAs (Figure 1A). This is confirmed both by functional and biochemical studies of individual protein-RNA interactions and by data on the proteins' cellular RNA interactomes. Indeed, data reporting on the RNA-binding landscape of RBPs has confirmed that they physically interact with hundreds, if not thousands of different RNA sites ${ }^{9}$.

The complexity and stratification of RNA regulation networks stems not only from the multiplicity of targets (and proteins binding to each target) but also from the multiple functions exerted by the individual proteins. Results on a number of well-studied, multi-functional RNA regulators (e.g. TIA- $1^{10,11}$, hnRNP A1 ${ }^{12,13,14}$, HuR ${ }^{15,16,17}, \mathrm{NF90}{ }^{18,19}$, Nucleolin ${ }^{20,21}$ and many others) indicate that the protein function is often target-dependent (Figure 1B). How strictly a function is associated to a set of targets has not yet been systematically investigated, but a relation has been validated in a small number of cases. For example, the ZBP1/IMP1/IGF2BP1 protein regulates the local translation of $\beta$-actin mRNA in neurons and fibroblasts, but does not appear to strongly regulate the stability of this mRNA $^{22}$. Instead, ZBP1 regulates the stability of mRNAs codifying for the CD44 and c-myc proteins in a range of cells ${ }^{23,24}$. A second example, among many, is the one of Lin28, a protein that regulates the maturation of Let-7 miRNA ${ }^{25}$. Lin 28 recruits a non-templated polymerase, TUT4, that adds a polyU tail to the 3' of the pre-miRNA, directing it to degradation ${ }^{26}$. However, Lin28 also plays a much more 
general role as a translational repressor ${ }^{27}$. Although the molecular basis for this function is still to be defined, it seems unlikely the recruitment of TUT4 and the poly-uridilation of the RNA target is part of this mechanism. These two examples highlight how the interaction of RNA regulatory proteins with different RNA partners is associated to both a different functional output, and a different molecular mechanism.

\section{Protein regulators recognise RNA targets using different arrangements of RNA binding domains}

As discussed above, the function of many RNA binding proteins depends on the target they are bound to. An important first step in understanding how protein-RNA interaction networks function is to define the molecular basis and the selectivity of the protein-RNA interactions. Eukaryotic RNA-binding proteins include RNA-binding domains (RBDs) that act as modules for RNA recognition, and provide a valuable key to decoding selectivity ${ }^{28}$. RBDs differ in size and specificity, and while many different RBDs exist, the most common RNA-binding domains: the RNA-recognition motif (RRM), hnRNPK-homology (KH) domain, double-stranded RNAbinding domain (dsRBD), zinc-finger ( $\mathrm{ZnF}$ ) motif, and cold shock domain (CSD) are found in tens to hundreds of functionally validated RNA binding proteins, representing a large share of the identified RNA-binding units in a cell ${ }^{7}$.

Recognised RNA sequences vary in their design: continuous or bipartite, short or extended, single-stranded or double-stranded, linear or complex e.g. contained within a stem loop. This generates diversity in target sequences, but also the necessity to be able to discern these different elements. Typically, RNA-binding proteins contain multiple RBDs, and it is the cooperation of different RBDs that provides the proteins with a toolkit for the recognition of these different features in the RNA targets (Figure 2A). For example, while individual sequence-specific RBDs typically recognise RNA sequences a few nucleotides long, it has been long established that inter-domain cooperation allows recognition of longer sequences as well as increasing the affinity of the interaction. Among the many examples, an early study showed how the two RRM domains of Drosophila Sex-lethal protein create a v-shaped unit to bind a 12-nucleotide long pyrimidine-rich $\operatorname{tract}^{29}$. It is worth mentioning that cooperativity in the binding of a longer RNA sequence can occur both in physically separated domains and in domains associated to form a rigid structure, as well as being associated with novel interdomain interactions ${ }^{28}$, as in the case of the recognition of a polyU tract by the splicing factor $\mathrm{U}_{2} \mathrm{AF}^{30}$. The multiple RBDs of a protein may also interact with sequences separated by a few non-interacting nucleotides, as observed for the RNA-editing enzyme ADAR2, whereby two independent dsRBDs separated by an 84-amino acid long linker recognise two distinct locations on a GluR-B mRNA stem loop ${ }^{31}$. In some cases, the interaction of short RNA sequences with individual domains of a multi-domain structure re-models the RNA structure. Examples include the KH3-KH4 di-domain of ZBP1 ${ }^{32}$, and the RRM3-RRM4 di-domain of polypyrimidine binding protein, where binding of the two domains to a polypyrimidine stretch in the proximity of a splice site is coupled to a looping of the RNA molecule that is thought to mediate alternative splicing events ${ }^{33}$. These few examples illustrate how RNA-binding proteins use multiple RNA-interacting domains in a range of combinatorial arrangements. It is worth mentioning that, although we describe above domains as stably folded and rigid units, RNA recognition is often associated to protein folding. This includes the folding of flexible peptide chains on an RNA structure, as is the case for the BIV Tat peptide binding to the RNA TAR structure $^{34}$, but also the rearrangement or stabilisation of secondary structure elements to trap the RNA target, such as in the UA1-RNA and p65-telomerase RNA complexes ${ }^{35,36}$, and the 
rearrangement or creation of inter-domain units, as observed with $\mathrm{U} 2 \mathrm{AF} 65^{37}$.

\section{The contribution of the individual RBDs to target recognition is often target-dependent}

An emerging point in the discussion on protein-RNA recognition is how the flexibility and different RNA-binding modes offered by multiple RBDs may be used by the same RNAbinding protein to recognise different targets. In the past ten years, a number of studies have shown that the roles played by individual domains of an RNA-binding protein in RNA recognition (and their structural arrangement) can vary, depending on the target (Figure 2B). This allows recognition of different sequences and structures, and for the protein to perform different molecular functions. For example, the small RNA regulator Lin28, which we introduced earlier as having functions in both miRNA maturation and translational control, encompasses two RNA-binding domains with different specificities and roles in recognition. A double CCHC ZnF motif recognises a GGAG RNA sequence with high sequence specificity $^{38}$, while a CSD has instead has been proposed to recognise a generic RNA hairpin structure with a moderate nucleobase bias for specific structural positions ${ }^{39}$. Lin28 recognition of the precursor of Let-7 miRNA is mediated by the recognition of a GGAG in the apical loop of the pre-miRNA by the $\mathrm{ZnF}$ di-domain, while the less specific CSD increases the affinity of the interaction. Unexpectedly, recent data on Lin28 regulation of the maturation of a neuronal miRNA, miR-9 $9^{40,41}$, show that in contrast to Let-7, the ZnF-GGAG interaction plays a lesser role in the interaction with this miRNA precursor. The difference between the two miRNAs seems to be linked to a different molecular mechanism, as degradation of miR-9 does not require binding of the TUT4 polymerase. A second example of different RNA binding modes for the same protein is the one of the splicing regulator PTB. PTB includes four RNA binding RRM domains, the last two of which, RRM3 and RRM4, create an inter-molecular two-domain structural unit ${ }^{33}$. Binding of PTB RRM3-RRM4 to the unstructured poly-pyrimidine tracts involved in the alternative splicing of $\mathrm{GABA}_{\mathrm{A}} \gamma 2$ pre-mRNA results in a looping of the RNA important for the functional mechanism ${ }^{42}$. Binding of RRM1 and RRM2 instead is thought to mainly stabilise the PTB-RNA interaction. However, in addition to regulating splicing, PTB binds to the highly-structured IRES RNA sequences from the poliovirus, picornavirus and encephalomyocarditis viruses, regulating viral mRNA translation ${ }^{43,44}$. Recent structural work indicates that the RRM domains of the protein recognise stem loops with the IRES complex RNA structure ${ }^{45}$. PTB structure and function are different from that of Lin28, and this example highlights how differential recognition is not limited to one type of RNA recognition module or cellular role.

These examples illustrate how different RNA recognition modes are associated to different regulatory processes. However, the domain-based recognition of different RNA targets can also be used to expand the reach of one regulatory mechanism. Recent work on the cancer factor RBM10 has shown that the interaction with a set of RNA targets containing a GGA sequence is mediated by two closely positioned RRM and $\mathrm{ZnF}$ domains. In addition, a second RRM domain selects for $\mathrm{C}$-rich sequences found in a second set of targets, including the cancerrelated Numb mRNA. Recognition of these targets does not mediate, in this case, a different function: protein binding to both GGA-containing and C-rich targets results in exon inclusion $^{46,47,48}$. Instead, the two recognition modes of the protein have been proposed to target regions on opposite sides of the intron-exon boundary with different nucleobase enrichment.

The proteins above are part of a growing ensemble of RNA regulators where multiple recognition modes expand protein function in RNA regulation. It is worth highlighting that the few RBDs discussed here include some of the most common RNA binding domains, i.e. RRM, $\mathrm{ZnF}, \mathrm{KH}$ and CSD (Figure 2), and are involved in a range of functions including regulating 
mRNA localisation and translational control, as well as in the metabolism of mRNAs and ncRNAs (Figure 1B). Target-dependent contribution of individual domains is widespread in RNA regulation.

\section{Isolating the contribution of individual RNA binding domains to the selection of the RNA targets is important to understanding the recognition of different targets}

The examples above challenge a model, whereby each protein and RNA-binding domain recognise the same recognition motif, or zipcode, on all of its physiological RNA targets, and explain that a target-specific use of the RBDs allows recognition of a diverse range of RNAs. Understanding the complexity of target recognition is necessary to relate the different regulatory functions performed by the protein, and to interpret global data on protein-RNA interaction and the functional output (e.g. on splicing and mRNA stability). However, evaluating the contribution of individual domains to the selection of the cellular targets is challenging. It requires both a structural understanding of the different binding modes to create accurate models of interaction, and a set of observables that report on RNA-binding of the protein in the cell, and ideally on protein function. Importantly, it also requires molecular tools to connect the different types of data.

Structure-informed conservative mutations that alter the affinity and specificity of individual RNA-binding domains can be used, at least in some cases, to de-convolute the contributions of the domains, and therefore the different RNA binding modes of an RBP. Point mutations have long been used to eliminate catalysis or RNA binding in helicases ${ }^{49,50}$. The recent structural insight into RNA recognition by a number of RNA-binding domains has helped extend this approach to investigate multi-domain protein-RNA recognition. The substantial body of knowledge available on the interaction of domains such as KH or RRM with RNA has identified residues that can be used to perturb RNA binding without changing structure, stability or, in principle, protein-protein interactions. For example, the roles of the individual domains of the protein KSRP in the interaction with mRNA and pre-miRNA have been examined using conservative point mutations in a RNA-interacting GXXG loop ${ }^{51}$. KSRP is a multi-functional protein that regulates different steps of mRNA metabolism, as well as the maturation of a small subset of miRNA. By testing the RNA binding of individual mutants, it was shown that the same domain plays a different role in the functional recognition of an AUrich and in the recognition of the pre-miRNA targets ${ }^{52,53,54}$. Interestingly, it was also found that a point mutation that changes the specificity, rather than the affinity of the interaction, impaired function and recognition - presumably because of competition with other RNA targets. That is, while the same domain can recognise different sequences in different targets, this does not reflect a lack of sequence preference at the structural level.

The same mutations that have been used to investigate the roles of individual domains in the recognition of individual targets can, in principle, be used in a more wide-range analysis of protein-RNA interactions. High-throughput sequencing has enabled a new generation of tools for the transcriptome-wide analysis of the interactions between a protein and the RNA targets both in vitro and in vivo ${ }^{54}$, including the powerful set of CLIP methods ${ }^{55,56,57,58}$. The results have revolutionised our understanding of RNA biology, revealing previously unknown protein functions and cell-specific complexities. However, these RNAseq-based methods have been less successful in resolving the ensembles of RNA targets that derive from different binding modes. A comparative analysis of CLIP data obtained from protein mutants with knocked out RNA binding in individual domains may allow us to directly relate the RNA-binding properties 
of a domain to target selection in the cell, and provide a powerful tool to de-convolute the different components of RNA binding, as well as extracting realistic binding modes that can be used in a broad set of analyses.

\section{Acknowledgements:}

\section{Declarations of interest:}

The Authors declare that there are no competing interests associated with the manuscript.

\section{Funding information:}

AR and GG are funded by MRC (MC_PC_13051) and UCL.

\section{References:}

1. Licatalosi, D. D., \& Darnell, R. B. (2010). RNA processing and its regulation: global insights into biological networks. Nature Reviews. Genetics, 11(1), 75-87.

2. Morris, A. R., Mukherjee, N., \& Keene, J. D. (2010), Systematic analysis of posttranscriptional gene expression. WIREs Syst Biol Med, 2(2), 162-180.

3. Glisovic, T., Bachorik, J. L., Yong, J., \& Dreyfuss, G. (2008). RNA-binding proteins and post-transcriptional gene regulation. FEBS Letters, 582(14), 1977-1986.

4. Kishore, S., Luber, S., \& Zavolan, M. (2010). Deciphering the role of RNA-binding proteins in the post-transcriptional control of gene expression. Briefings in Functional Genomics, 9(5-6), 391-404.

5. Hasan, A., Cotobal, C., Duncan, C. D. S., \& Mata, J. (2014). Systematic Analysis of the Role of RNA-Binding Proteins in the Regulation of RNA Stability. PLoS Genetics, 10(11).

6. Besse, F., \& Ephrussi, A. (2008). Translational control of localized mRNAs: restricting protein synthesis in space and time. Nature Reviews Molecular Cell Biology, 9(12), 971-980.

7. Gerstberger, S., Hafner, M., \& Tuschl, T. (2014). A census of human RNA-binding proteins. Nature Publishing Group, 15(12), 829-845.

8. Marinov, K.G., Williams, B.A., McCue, K., Schroth, G.P., Gertz, J., Myers, G.M. \& Wold, B.J. (2014) From single-cell to cell-pool transcriptomes: Stochasticity in gene expression and RNA splicing. Genome Res. 24(3): 496-510

9. Ascano, M., Hafner, M., Cekan P., Gerstberger S \&Tuschl T. (2012). Identification of RNA-protein interaction networks using PAR-CLIP.Wiley Interdiscip Rev RNA. 3(2):159-77.

10. Zhao, W., Zhao, J., Hou, M., Wang, Y., Zhang, Y., Zhao, X., Zhang, C., \& Guo, D. (2014). HuR and TIA1/TIAL1 are involved in regulation of alternative splicing of SIRT1 pre-mRNA. International Journal of Molecular Sciences, 15(2), 2946-2958.

11. Damgaard, C. K., \& Lykke-Andersen, J. (2011). Translational coregulation of 5'TOP mRNAs by TIA-1 and TIAR. Genes and Development, 25(19), 2057-2068. 
12. Zhao, T. T., Graber, T. E., Jordan, L. E., Cloutier, M., Lewis, S. M., Goulet, I., Côté., \& Holcik, M. (2009). hnRNP A1 regulates UV-induced NF-kappaB signalling through destabilization of cIAP1 mRNA. Cell Death and Differentiation, 16(2), 244252.

13. Roy, R., Durie, D., Li, H., Liu, B. Q., Skehel, J. M. ark, Mauri, F., Cuorvo, L. V., Barbareschi, M., Guo, L., Holcik, M., Seckl, M. J., \& Pardo, O. E. (2014). hnRNPA1 couples nuclear export and translation of specific mRNAs downstream of FGF2/S6K2 signalling. Nucleic Acids Research, 42(20), 12483-12497.

14. Guo, R., Li, Y., Ning, J., Sun, D., Lin, L., \& Liu, X. (2013). HnRNP A1/A2 and SF2/ASF Regulate Alternative Splicing of Interferon Regulatory Factor-3 and Affect Immunomodulatory Functions in Human Non-Small Cell Lung Cancer Cells. PLoS ONE, 8(4), 11-13.

15. Popovitchenko, T., Thompson, K., Viljetic, B., Jiao, X., Kontonyiannis, D. L., Kiledjian, M., Hart, R. P., \& Rasin, M. R. (2016). The RNA binding protein HuR determines the differential translation of autism-associated FoxP subfamily members in the developing neocortex. Scientific Reports, 6(1), 28998.

16. Chang, N., Yi, J., Guo, G., Liu, X., Shang, Y., Tong, T., Cui, Q., Zhan, M., Gorospe, M., \& Wang, W. (2010). HuR Uses AUF1 as a Cofactor To Promote p16INK4 mRNA Decay. Molecular and Cellular Biology, 30(15), 3875-3886.

17. Xu, Y. Z., Marco, S. D., Gallouzi, I., Rola-Pleszczynski, M., \& Radzioch, D. (2005). RNA-Binding Protein HuR Is Required for Stabilization of. Molecular and Cellular Biology, 25(18), 8139-8149.

18. Li, Y., Masaki, T., Shimakami, T., \& Lemon, S. M. (2014). hnRNP L and NF90 Interact with Hepatitis C Virus 5'-Terminal Untranslated RNA and Promote Efficient Replication. Journal of Virology, 88(13), 7199-7209.

19. Kuwano, Y., Pullmann, R., Marasa, B. S., Abdelmohsen, K., Lee, E. K., Yang, X., Martindale, J. L., Zhan, M., \& Gorospe, M. (2009). NF90 selectively represses the translation of target mRNAs bearing an AU-rich signature motif. Nucleic Acids Research, 38(1), 225-238.

20. Saha, S., Chakraborty, A., \& Bandyopadhyay, S. S. (2016). Stabilization of Oncostatin-M mRNA by Binding of Nucleolin to a GC-Rich Element in Its 3'UTR. Journal of Cellular Biochemistry, 117(4), 988-999.

21. Abdelmohsen, K., Tominaga, K., Lee, E. K., Srikantan, S., Kang, M. J., Kim, M. M., Selimyan, R., Martindale, J. L., Yang, X., Carrier, F., Zhan, M., Becker, K. G., \& Gorospe, M. (2011). Enhanced translation by Nucleolin via G-rich elements in coding and non-coding regions of target mRNAs. Nucleic Acids Research, 39(19), 85138530.

22. Patel, V. L., Mitra, S., Harris, R., Buxbaum, A. R., Lionnet, T., Brenowitz, M., Girvin, M., Levy, M., Almo, S. C., Singer, R. H., \& Chao, J. A. (2012). Spatial arrangement of an RNA zipcode identifies mRNAs under post-transcriptional control. Genes and Development, 26(1), 43-53.

23. Noubissi, F. K., Elcheva, I., Bhatia, N., Shakoori, A., Ougolkov, A., Liu, J., Minamoto, T., Ross, J., Fuchs, S. Y., \& Spiegelman, V. S. (2006). CRD-BP mediates stabilization of betaTrCP1 and c-myc mRNA in response to beta-catenin signalling. Nature, 441(7095), 898-901.

24. Vikesaa, J., Vo Hansen, T., Jonson, L., Borup, R., Wewer, U. M., Christiansen, J., \& Nielsen, F. C. (2006). RNA-binding IMPs promote cell adhesion and invadopodia formation. The EMBO Journal, 25(7)

25. Heo, I., Joo, C., Cho, J., Ha, M., Han, J., \& Kim, V. N. (2008). Lin 28 Mediates the Terminal Uridylation of let-7 Precursor MicroRNA. Molecular Cell, 32(2), 276-284. 
26. Heo, I., Joo, C., Kim, Y. K., Ha, M., Yoon, M. J., Cho, J., Yeom, K. H., Han, J., \& Kim, V. N. (2009). TUT4 in Concert with Lin28 Suppresses MicroRNA Biogenesis through Pre-MicroRNA Uridylation. Cell, 138(4), 696-708.

27. Cho, J., Chang, H., Kwon, S. C., Kim, B., Kim, Y., Choe, J., Ha, M., \& Kim, V. N. (2012). LIN28A is a suppressor of ER-associated translation in embryonic stem cells. Cell, 151(4), 765-777.

28. Lunde, B. M., Moore, C., \& Varani, G. (2007). RNA-binding proteins: modular design for efficient function. Nature Reviews. Molecular Cell Biology, 8(6), 479-90.

29. Handa, N., Nureki, O., Kurimoto, K., Kim, I., Sakamoto, H., Shimura, Y., Muto, Y., \& Yokoyama, S. (1999). Structural basis for recognition of the tra mRNA precursor by the Sex-lethal protein. Nature, 398(6728), 579-585.

30. Mackereth CD, Madl T, Bonnal S, Simon B, Zanier K, Gasch A, Rybin V, Valcárcel J, Sattler M. (2011). Multi-domain conformational selection underlies pre-mRNA splicing regulation by U2AF. Nature, 475(7356):408-11.

31. Stefl, R., Xu, M., Skrisovska, L., Emeson, R. B., \& Allain, F. H. T. (2006). Structure and specific RNA binding of ADAR2 double-stranded RNA binding motifs. Structure, 14(2), 345-355.

32. Chao, J. A., Patskovsky, Y., Patel, V., Levy, M., Almo, S. C., \& Singer, R. H. (2010). ZBP1 recognition of $\beta$-actin zipcode induces RNA looping. Genes and Development, 24(2), 148-158.

33. Oberstrass, F. C., Auweter, S. D., Erat, M., Hargous, Y., Henning, A., Wenter, P., Reymond, L., Amir-Ahmady, B., Pitsch, S., Black, D. L., \& Allain, F. H.-T. (2005). Structure of PTB Bound to RNA: Specific Binding and Implications for Splicing Regulation. Science, 309(5743), 2054-2057.

34. Puglisi, J. D., Chen, L., Blanchard, S., \& Frankel, A. D. (1995). Solution Structure of a Bovine Immunodeficiency Virus Tat-TAR Peptide-RNA Complex. Science, 270(5239), 1200-1203.

35. Allain, F. H.-T., Gubser, C. C., Howe, P. W. A., Nagai, K., Neuhaus, D., \& Varani, G. (1996). Specificity of ribonucleoprotein interaction determined by RNA folding during complex formation. Nature, 380(6575), 646-650.

36. Singh, M., Wang, Z., Koo, B. K., Patel, A., Cascio, D., Collins, K., \& Feigon, J. (2012). Structural Basis for Telomerase RNA Recognition and RNP Assembly by the Holoenzyme La Family Protein p65. Molecular Cell, 47(1), 16-26.

37. Mackereth, C. D., Madl, T., Bonnal, S., Simon, B., Zanier, K., Gasch, A., Rybin, V., Valcarcel, J., \& Sattler, M. (2011). Multi-domain conformational selection underlies pre-mRNA splicing regulation by U2AF. Nature, 475(7356), 408-411.

38. Loughlin, F. E., Gebert, L. F. R., Towbin, H., Brunschweiger, A., Hall, J., \& Allain, F. H.-T. (2011). Structural basis of pre-let-7 miRNA recognition by the zinc knuckles of pluripotency factor Lin28. Nature Structural \& Molecular Biology, 19(1), 84-89.

39. Nam, Y., Chen, C., Gregory, R. I., Chou, J. J., \& Sliz, P. (2011). Molecular basis for interaction of let-7 MicroRNAs with Lin28. Cell, 147(5), 1080-1091.

40. Nowak, J. S., Choudhury, N. R., de Lima Alves, F., Rappsilber, J., \& Michlewski, G. (2014). Lin28a regulates neuronal differentiation and controls miR-9 production. Nature Communications, 5, 3687.

41. Nowak, J. S., Hobor, F., Downie Ruiz Velasco, A., Choudhury, N. R., Heikel, G., Kerr, A., Ramos, A., \& Michlewski, G. (2016). Lin28a uses distinct mechanisms of binding to RNA and affects positively and negatively miRNA levels. RNA (New York, N.Y.), 23(3), 317-332. 
42. Wagner, E. J., \& Garcia-Blanco, M. A. (2001). Polypyrimidine Tract Binding Protein Antagonizes Exon Definition. Molecular and Cellular Biology, 21(10), 3281-3288.

43. Kafasla, P., Morgner, N., Pöyry, T. A. A., Curry, S., Robinson, C. V., \& Jackson, R. J. (2009). Polypyrimidine Tract Binding Protein Stabilizes the Encephalomyocarditis Virus IRES Structure via Binding Multiple Sites in a Unique Orientation. Molecular Cell, 34(5), 556-568.

44. Kafasla, P., Morgner, N., Robinson, C. V, \& Jackson, R. J. (2010). Polypyrimidine tract-binding protein stimulates the poliovirus IRES by modulating eIF4G binding. The EMBO Journal, 29(21), 3710-22.

45. Dorn, G., Leitner, A., Boudet, J., Campagne, S., von Schroetter, C., Moursy, A., Aebersold, \& R., Allain, F. H.-T. (2017). Structural modeling of protein-RNA complexes using crosslinking of segmentally isotope-labeled RNA and MS/MS. Nat Meth, 14(5), 487-490.

46. Collins, K. M., Kainov, Y. A., Christodolou, E., Ray, D., Morris, Q., Hughes, T., Taylor, I. A., Makeyev, E. V., \& Ramos, A. (2017). An RRM-ZnF RNA recognition module targets RBM10 to exonic sequences to promote exon exclusion. Nucleic Acids Research, 14(11), 6761-6774.

47. Bechara, E. G., Sebestyen, E., Bernardis, I., Eyras, E., \& Valcarcel, J. (2013). RBM5, 6 , and 10 differentially regulate NUMB alternative splicing to control cancer cell proliferation. Molecular Cell, 52(5), 720-733.

48. Hernandez, J., Bechara, E., Schlesinger, D., Delgado, J., Serrano, L., \& Valcarcel, J. (2016). Tumor suppressor properties of the splicing regulatory factor RBM10. RNA Biology, 13(4), 466-472.

49. Heilek, G. M., \& Peterson, M. G. (1997). A point mutation abolishes the helicase but not the nucleoside triphosphatse activity of hepatitis $\mathrm{C}$ virus ns 3 protein. $J$ Virol, 71(8), 6264-6266.

50. Nicklas, S., Okawa, S., Hillje, A. L., Gonzalez-Cano, L., Sol, A. Del, \& Schwamborn, J. C. (2015). The RNA helicase DDX6 regulates cell-fate specification in neural stem cells via miRNAs. Nucleic Acids Research, 43(5), 2638-2654.

51. Hollingworth, D., Candel, A. M., Nicastro, G., Martin, S. R., Briata, P., Gherzi, R., \& Ramos, A. (2012). KH domains with impaired nucleic acid binding as a tool for functional analysis. Nucleic Acids Research, 40(14), 6873-6886.

52. Gherzi, R., Lee, K. Y., Briata, P., Wegmüller, D., Moroni, C., Karin, M., \& Chen, C. Y. (2004). A KH domain RNA binding protein, KSRP, promotes ARE-directed mRNA turnover by recruiting the degradation machinery. Molecular Cell, 14(5), 571583.

53. Garcia-Mayoral, M. F., Hollingworth, D., Masino, L., Diaz-Moreno, I., Kelly, G., Gherzi, R., Chou, C. F., Chen, C. Y., \& Ramos, A. (2007). The Structure of the CTerminal KH Domains of KSRP Reveals a Noncanonical Motif Important for mRNA Degradation. Structure, 15(4), 485-498.

54. Garcia-Mayoral, M. F., Diaz-Moreno, I., Hollingworth, D., \& Ramos, A. (2008). The sequence selectivity of KSRP explains its flexibility in the recognition of the RNA targets. Nucleic Acids Research, 36(16), 5290-5296.

55. Campbell, Z.T. \& Wickens, M. (2016) Probing RNA-protein networks: biochemistry meets genomics. Trends Biochem Sci., 40(3), 157-164.

56. Ule, J., Jensen, K. B., Ruggiu, M., Mele, A., Ule, A., \& Darnell, R. B. (2003). CLIP Identifies Nova-Regulated RNA Networks in the Brain. Science, 302(5648), 12121215.

57. Hafner, M., Landthaler, M., Burger, L., Khorshid, M., Hausser, J., Berninger, P., Rothballer, A., Ascano, M., Jungkamp, A., Munschauer, M., Ulrich, A., Wardle, G. 
S., Dewell, S., Zavolan, \& M., Tuschl, T. (2010). Transcriptome-wide Identification of RNA-Binding Protein and MicroRNA Target Sites by PAR-CLIP. Cell, 141(1), 129-141.

58. Tollervey, J. R., Curk, T., Rogelj, B., Briese, M., Cereda, M., Kayikci, M., König, J., Hortobágyi, T., Nishimura, A. L., Župunski, V., Patani, R., Chandran, S., Rot, G., Zupan, B., Shaw, C. E., \& Ule, J. (2011). Characterizing the RNA targets and position-dependent splicing regulation by TDP-43. Nature Neuroscience, 14(4), 452458.

\section{Figure Legend:}

Figure 1 - RNA-binding proteins recognise multiple RNA targets, and exert multiple functions in a target-dependent manner. (A) Simple diagram highlighting that RBPs frequently have numerous, and sometimes overlapping, RNA targets.(B) The multiple functions of a representative group of well-studied of RNA degradation is summarised here.

Figure 2 - Combinatorial usage of RBDs allows for multiple RNA-binding modes, expanding an RBPs target repertoire. (A) From top to bottom: RBDs cooperate to recognise the target RNAs. They are also capable of recognising sequences separated by multiple non-interacting nucleotides, or belonging to different RNA molecules entirely. RBDs can recruit additional proteins to an RNA. Lastly, RBDs can interact with multiple sequences within a target to remodel the bound RNA.(B) RBDs can use their multiple domains in a target-dependent fashion. Here we summarise the contribution of the individual domains of six representative of RBDs to the overall binding of each protein. Straight lines indicate marginal or unclear contributions, and the width of the triangle represents the relative importance of the contributions with respect to overall binding. 
A

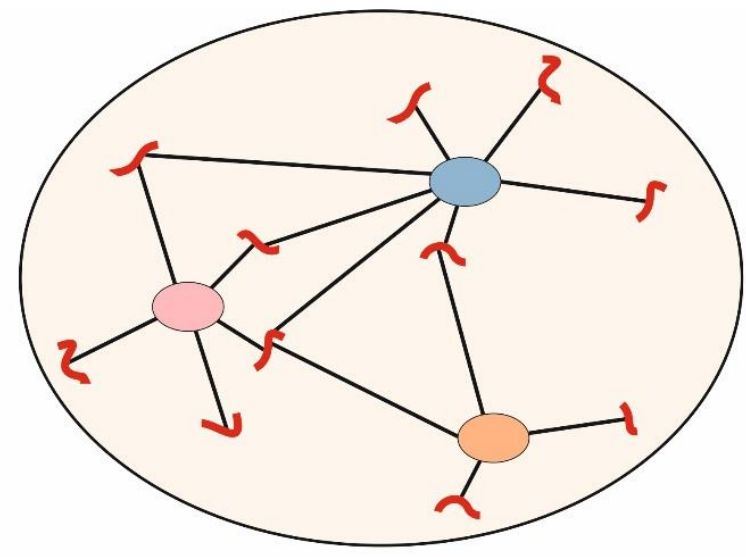

B
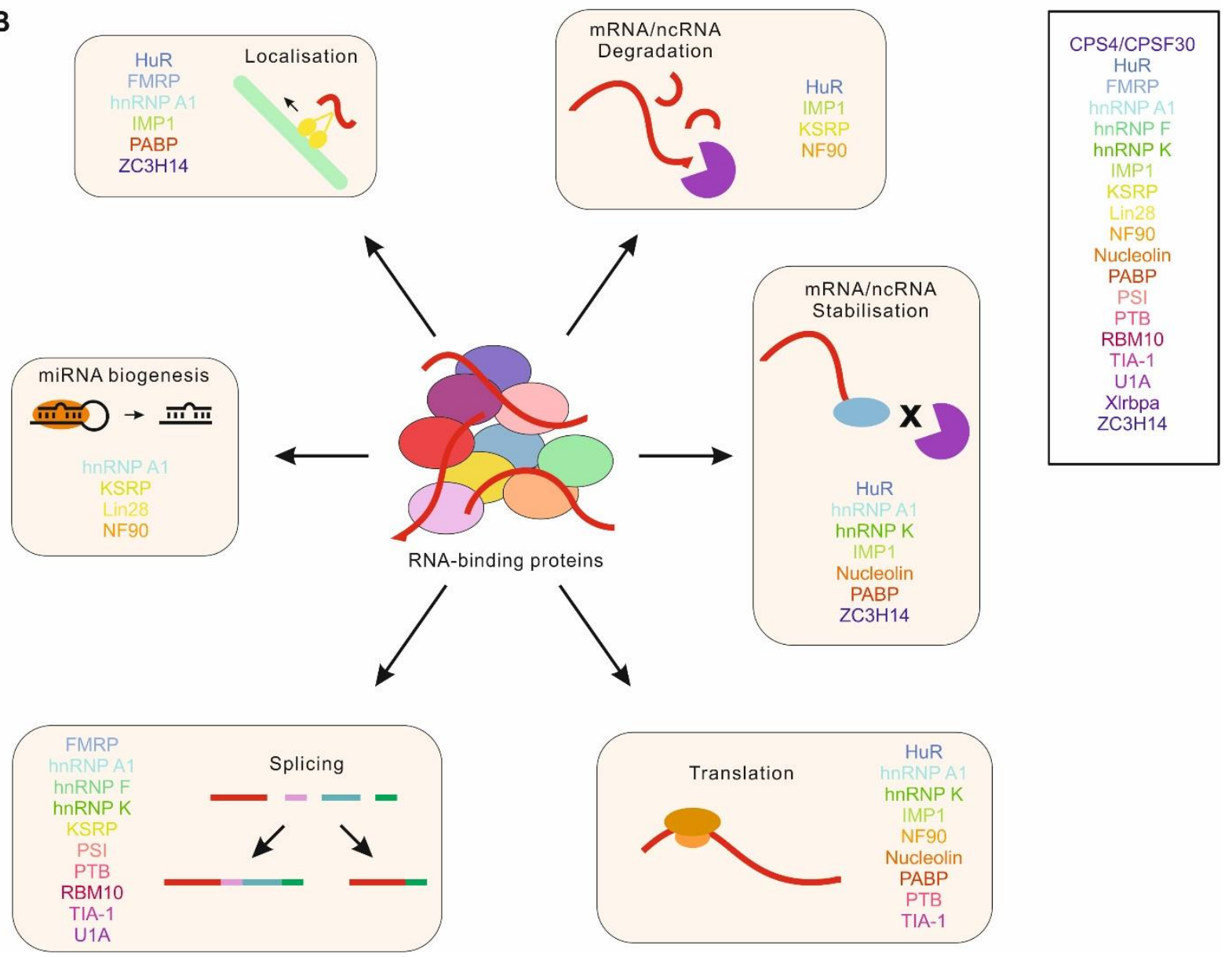

CPS4/CPSF 30

HuR

FMRP

hnRNP F

hnRNP K

IMP1

KSTP
$\operatorname{Lin} 28$

RBM1

Xlrbpa

ZC3H14 
A
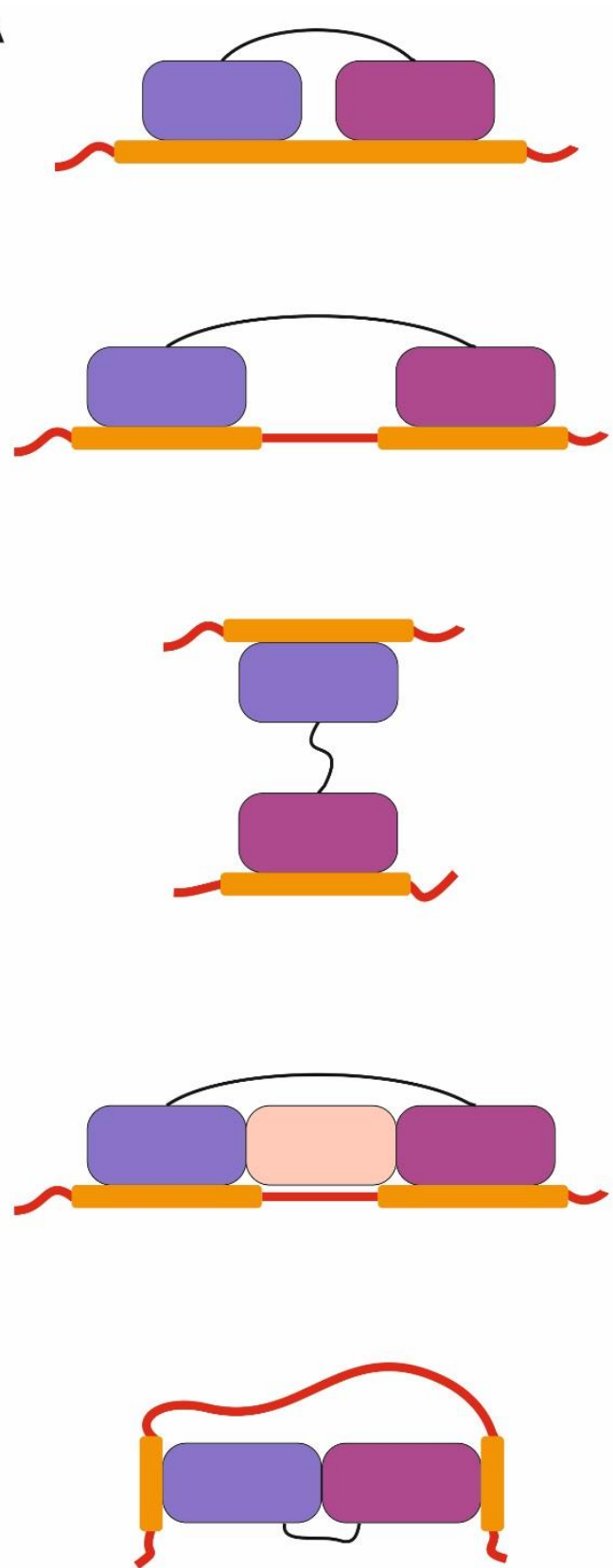

B

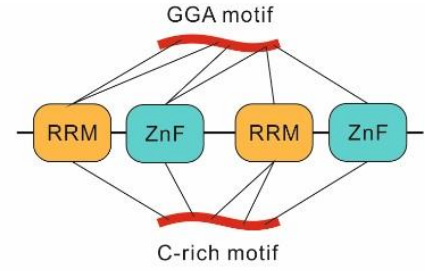

NF90
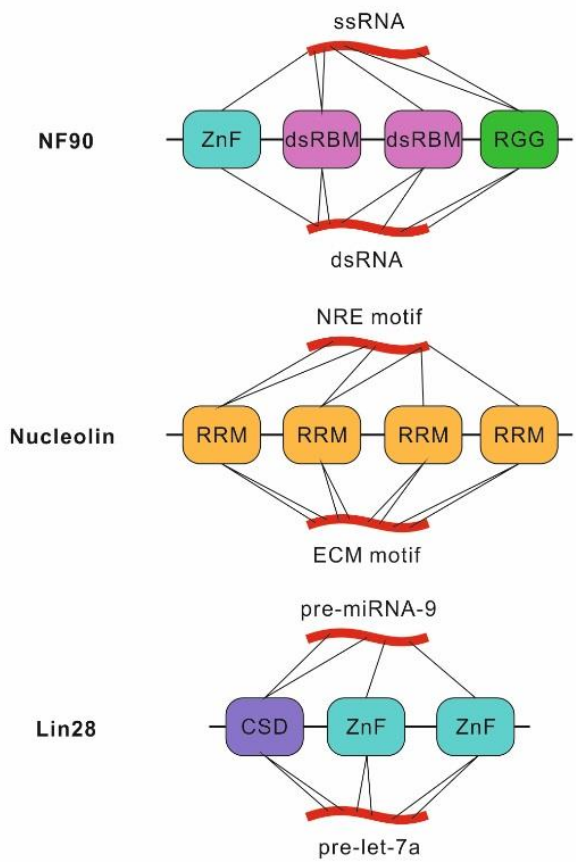

b-actin mRNA

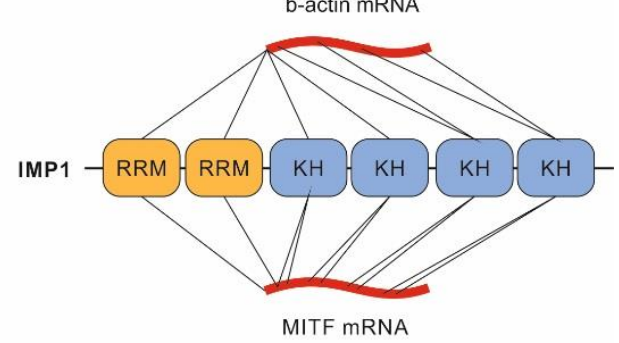

EMCV IRES

Ртв

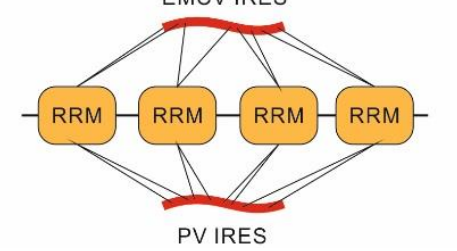

